\title{
Research center of biomanufacturing in Xi'an Jiaotong University
}

\author{
Ling Wang ${ }^{1,2} \cdot$ Dichen $\mathrm{Li}^{1,2} \cdot$ Jiankang $\mathrm{He}^{1,2} \cdot$ Bingheng $\mathrm{Lu}^{1,2}$ \\ Received: 22 October 2018 / Accepted: 27 October 2018 / Published online: 29 November 2018 \\ (c) Zhejiang University Press 2018
}

\section{Overview}

Xi'an Jiaotong University (XJTU) has carried out the research of additive manufacturing (AM) since 1993, who is one of the earliest institutes majoring in AM. After 20 years of effort, XJTU has made great progress on the additive manufacturing of polymer, metals, ceramics, composite materials and intelligent materials. XJTU has established a research team that features the engineering application of rapid manufacturing system. A Ministry of Education's inno- vative team, which is academically leaded by Prof. Bingheng $\mathrm{Lu}$, was formed in the field of AM. The basic research was supported by the State Key Laboratory of Manufacturing System Engineering (XJTU). XJTU has won a second prize of the State Award for Scientific and Technological Progress, three second prizes of State Award for Technological Invention and four first prizes of ministerial and provincial science and technology award in the field of AM. XJTU has more than 300 invention patents about AM.
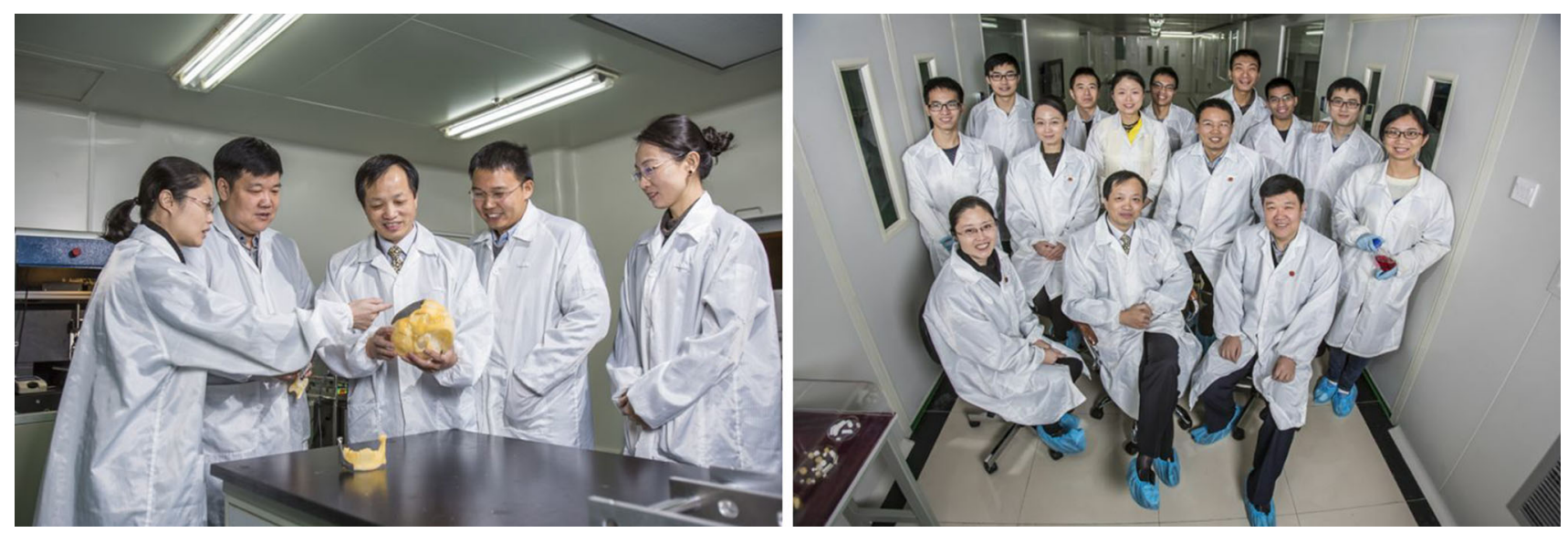

(a) Research staff From left to right: Associate Prof. Qin Lian, Prof. Yaxiong Liu, Prof. Li, Prof. Jiankang He, Associate Prof. Ling Wang;

(b) Prof. Li and partially the group member in the lab.

Ling Wang

menlwang@mail.xjtu.edu.cn

1 State Key Lab for Manufacturing System Engineering, Xi' an Jiaotong University, Xi' an 710054, China

2 School of Mechanical Engineering, Xi' an Jiaotong University, Xi' an 710049, China
Supported by National Natural Science Foundation (NSFC) of China, XJTU took the lead in the development of biofabrication. The first customized mandibular substitute surgery in the world was carried out by XJTU in 2001. In the research of customized bone substitutes, the problem of personalized precise adaptation was solved, and an in situ design methodology was proposed for customized craniomaxillofacial substitutes to promote bone in-growth. XJTU developed an integrated technology of bionic design and manufacturing technology for craniomaxillofacial substitutes. Based on this technology, specialized 3D printing equipment has been developed and commercialized, thousands of clinical cases 
have been carried out, and good physiological functions of patients have been restored. The research achievement on 'Design and Manufacturing Technology of Customized Craniomaxillofacial Implants' has won the second prize of State Award for Technological Invention in 2014 and the golden prize of the first 'Good Design in China' in 2015.

XJTU has also carried out the research on 3D printing of polyetheretherketone (PEEK) orthopedic implants and implemented the world's first clinical trial of 3D-printed PEEK rib and heart plate implants. Focusing on the controllable fabrication of the typical soft and hard tissue of the human body (liver, bone), the microvascular gradient the high-performance customized orthopedic implants. The team has developed a 3D printing technology of propertycontrolled cold deposition and the corresponding intelligent process, which can produce PEEK component with adjustable properties (stiffness and modulus). Based on that, the rapid manufacturing of high-performance, low-cost, high-precision PEEK orthopedic implants can be achieved. The relative techniques take the leading position world widely, and the clinical application was implemented in Xi' an in 2017, noted as the world's first 3D-printed PEEK ribs. Moreover, the team has won the second prize at Solvay AM Cup for the best precision of printing [1].
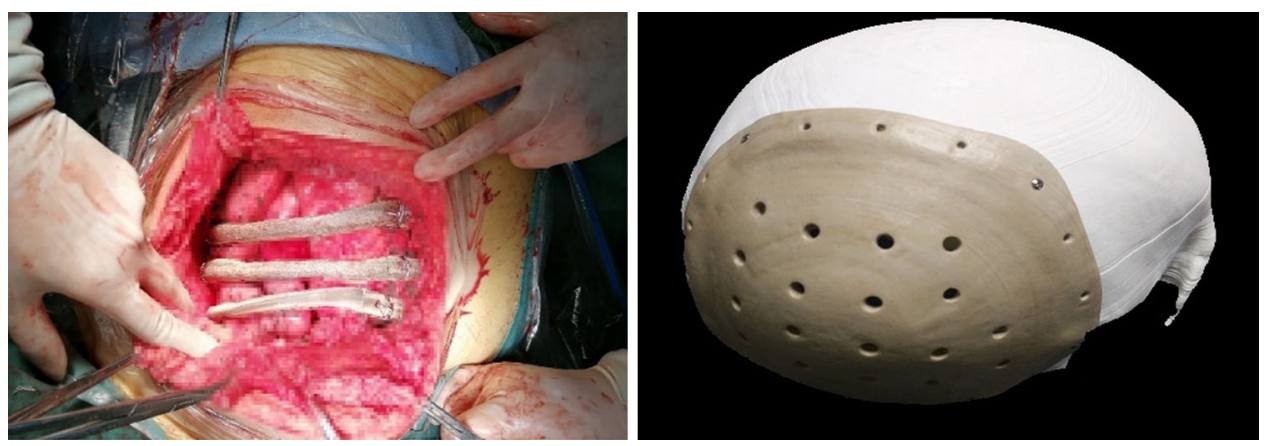

(a) The world's first clinical application on 3D-printed PEEK rib; (b) 3D-printed PEEK skull prosthesis

structure of soft and hard tissue fixation interface (ligament/cartilage/bone), systematic research work was carried out at three levels: the theoretical basis of bionic design, the additive manufacturing method of macro-/microstructure and the mechanisms of microstructure promoting tissue regeneration. Moreover, the $4 \mathrm{D}$ printing technology has been developed for the biofabricate of degradable tracheal stent and breast scaffold implant and the first international clinical trial was implemented, which stimulated the development of biofabricating technology of biodegradable engineering tissue and organ develops from benchwork to clinical applications. The academic achievements were quoted and positively evaluated by many international scholars. Finally, XJTU has achieved the very first CFDA registration certificate of customized implant in China, which is a milestone in the industrialization of 3D bioprinting technology in China.

\section{Research directions}

\section{Design and 3D printing technology of customized PEEK orthopedic implant}

The 3D printing technology of PEEK is based on the biomaterials of polyetheretherketone and was used to manufacture

\section{Software developed for customized orthopedic implants (OrthoDesign)}

To solve the existed problem of the current designing methodology for the design of customized prosthesis, lack of the ability of biomechanical analysis and the standard operational procedure (SOP), a software was developed by integrating five modulus of: reconstruction of the bone, implants, bone/prosthesis assembly, optimization analysis, and microstructural modeling, to realize the rapidly intelligent design in both of the macro-level (geometry) and the micro-level (microstructural design) of customized medical prosthesis. The CAD file of the designed prosthesis can be directly used for 3D printing manufacturing. Most importantly, the optimized microstructural design can improve the long-term stability of the implants with excellent bone ingrowth [2-6]. 


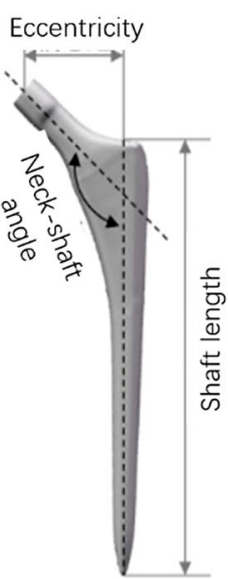

Key size design

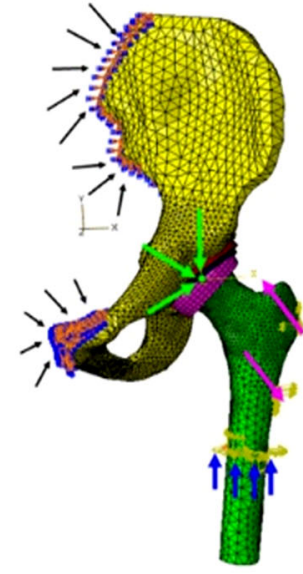

Performance analysis of prosthesis after implantation

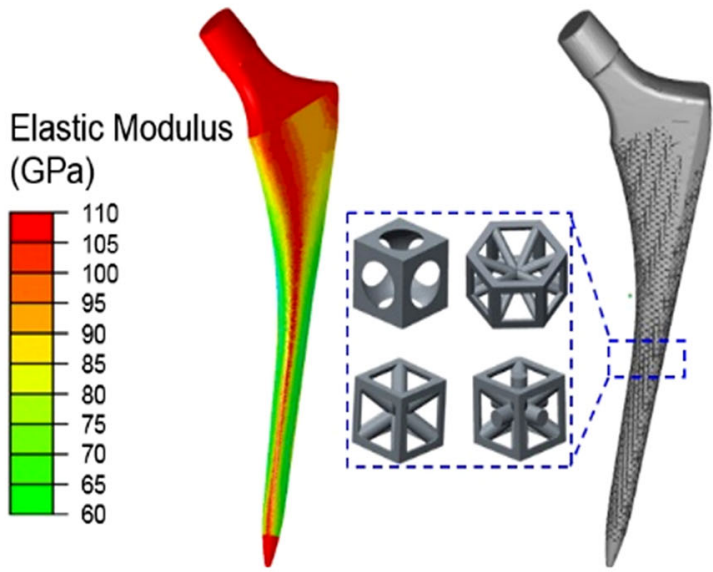

Results of elastic optimization The porous STL model of femoral shaft of femoral shaft

Procedure of Orthodesign for the design of customized prosthesis: hip femoral stem

\section{D printing technology for repairing articular cartilage defects}

This technique aims for repairing the large-scale bone/cartilage defects, based on the intra-articular biomechanics and biological environment of the joint, to meet the demands of designing, manufacturing and functional evaluation for multi-material and multi-level bone/cartilage scaffold. The development of manufacturing technology and functional evaluation technique for bone/cartilage substitute has provided good scientific foundation and engineering implementation for the biofabrication of artificial living joint tissue with improved mechanical and biological properties [7-16].

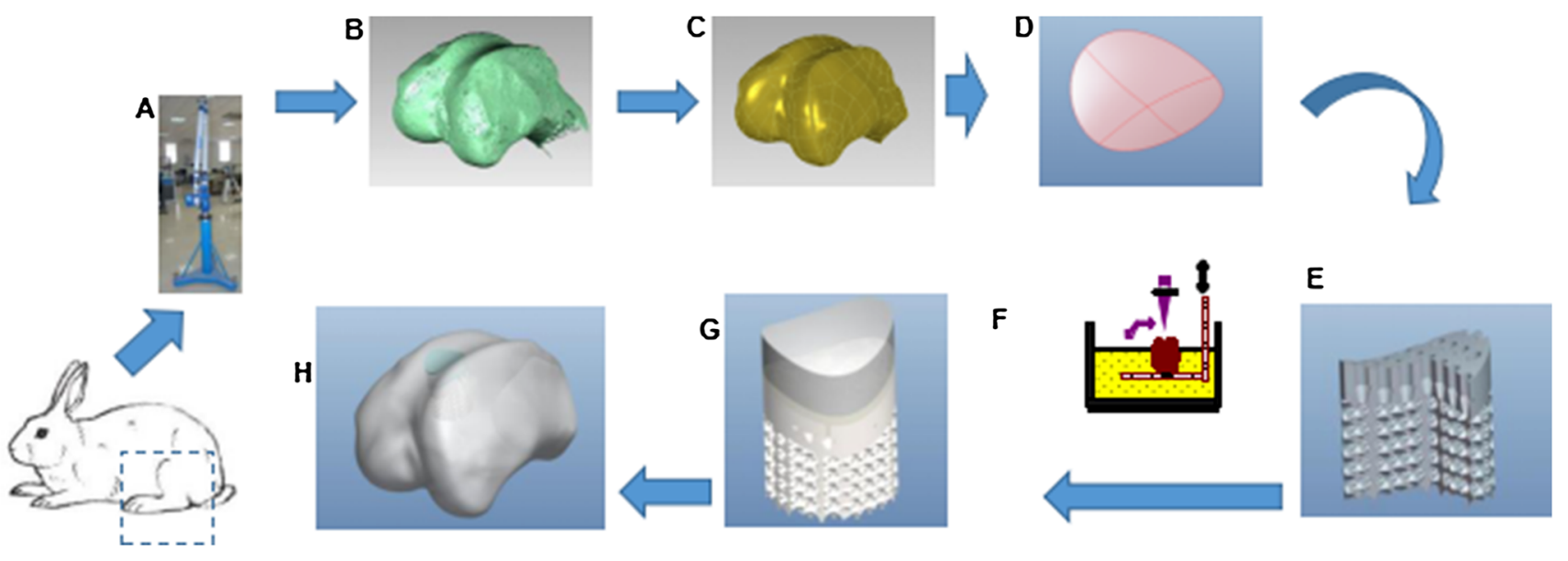

Procedure of design and manufacturing of large-size bone/cartilage scaffold 
A precise stereolithography (SLA) additive manufacturing technology was developed in this group for the biofabrication of multi-material and multi-scale scaffold, and the experimental platform and equipment were built in-house for manufacturing of large-scale bionic scaffold. The equipment is developed with a new printing processing system of SLA ceramic slurry, to realize a continuous printing technique with no obvious layer structure. At present, this technology has an automatic feedback strategy to control the printing defects during the forming process, based on that two sets of complete $3 \mathrm{D}$ printing processes and the corresponding experimental prototypes were developed allowing the fast manufacturing of bionic scaffolds with high precision and complex geometry at the maximum printing size of $30 \mathrm{~mm} \times$ $30 \mathrm{~mm} \times 30 \mathrm{~mm}$. An in vitro culture and functional evaluation system has been developed to adopt the biomechanical and physiological environment of joint. Animal studies (rabbits, dogs and sheep) were also carried out to validate the effectiveness of the design and manufacturing for osteochondral composite scaffolds.

\section{Biofabrication and clinical application of biodegradable customize implant}

The team also majored in the 3D printing of biodegradable materials for the application of breast tissue replacement and tracheal scaffold. The system of bionic design and 3D printing technology has been established for biofabrication of flexible biodegradable soft tissue scaffold, and collaborated with Xijing Hospital of the Fourth Military Medical University, the first international clinical trial of a customized biodegradable flexible breast stent was implemented. Up to date, 14 clinical cases have been implemented with good clinical outcome. The implanted stent has formed good match to the morphology and mechanical properties of the host tissue, additionally, good self-repair mechanisms has also been achieved through the process of host tissue regeneration and scaffold degradation. This technique provides a novel way for breast-conserving treatment of breast cancer [17-25].
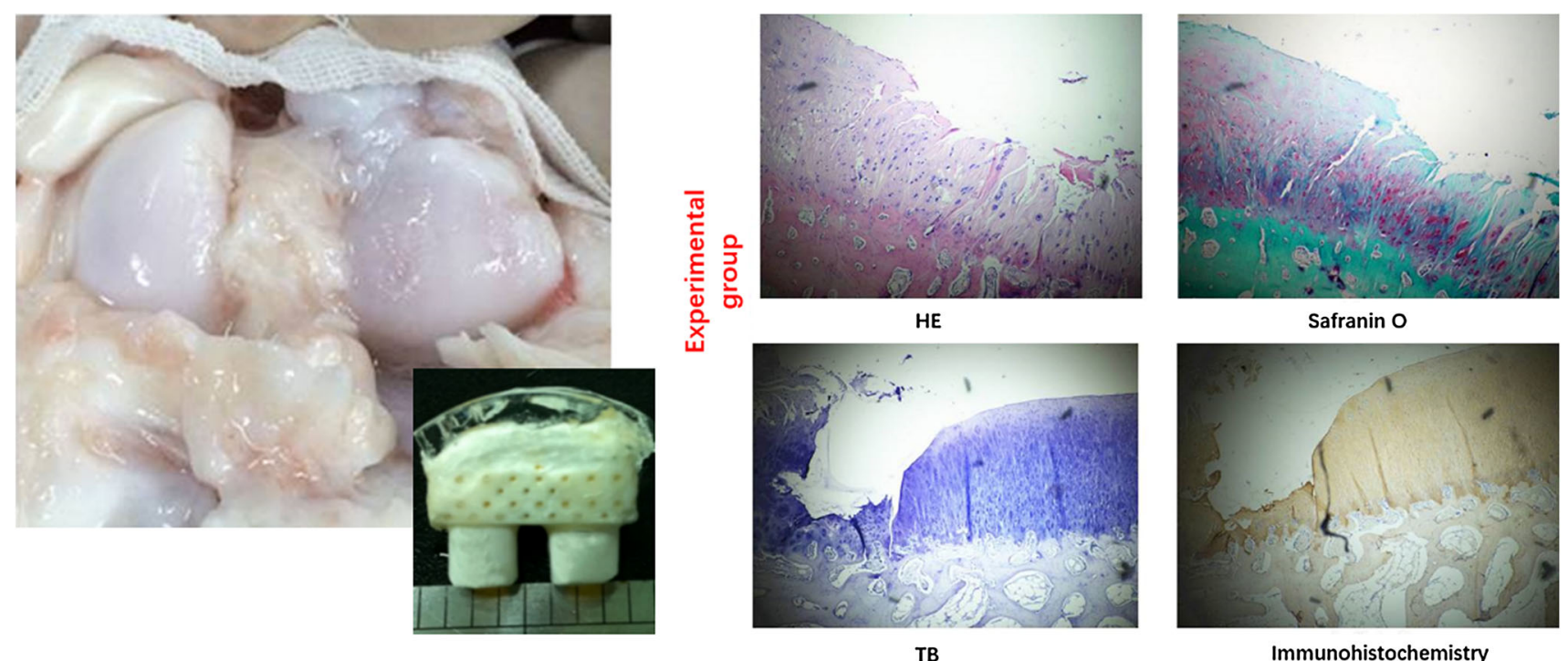

Immunohistochemistry

Animal test of large-scale bone/cartilage defect repair (the picture on the left shows general observation of the sheep knee joint after implantation of 6 months, and the right one shows histological staining of the implanted scaffold) 

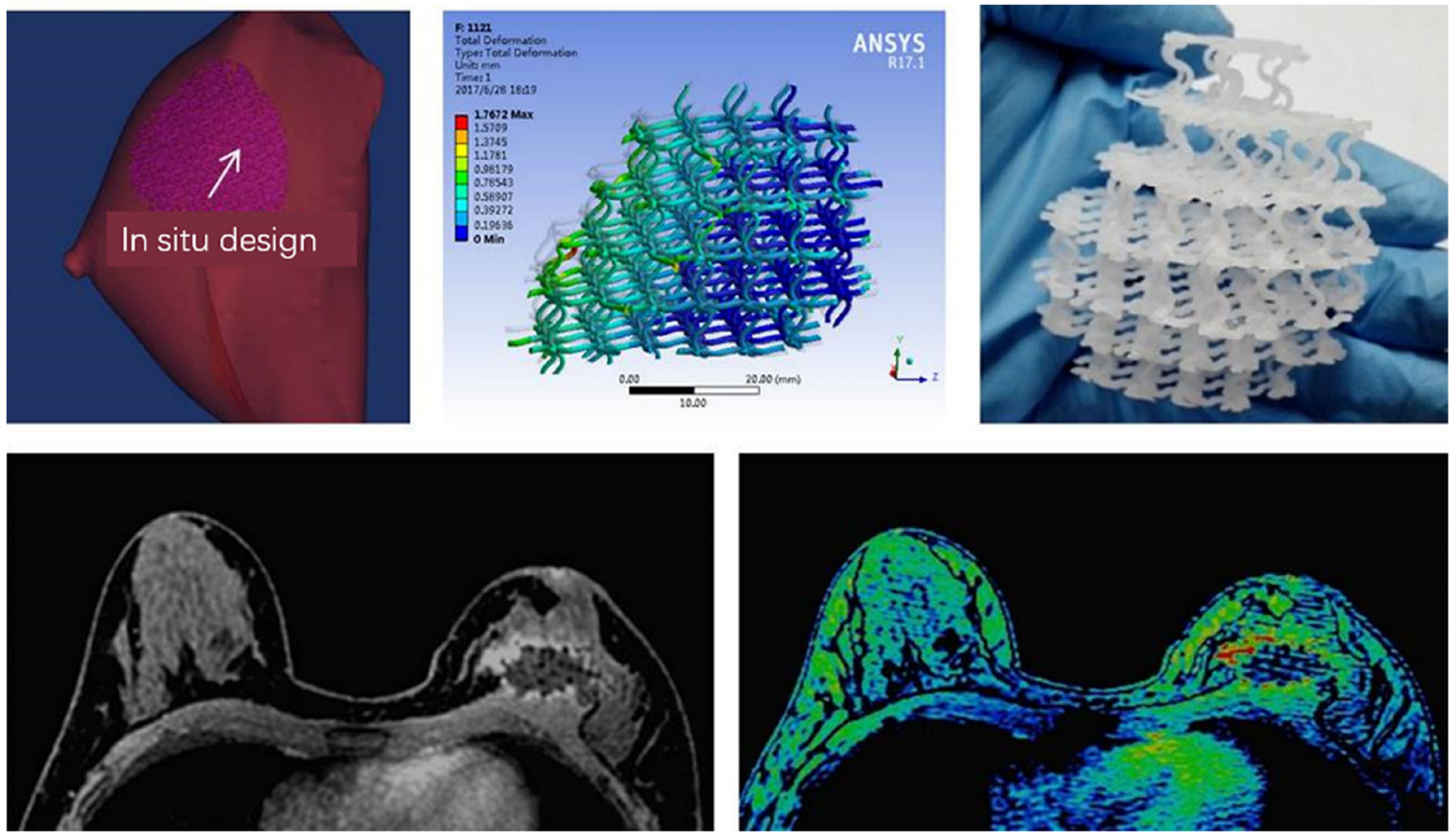

Design, manufacture and clinical application of personalized biodegradable breast scaffold

The team has also proposed a tracheal suspension surgery based on the printed biodegradable tracheal stent and has the first clinical application in China. Up until now, 4 clinical trials have been implemented with good clinical performance.
Clinical trials were reported by CCTV7, CCTV10, Xinhua News Agency, People's Daily and other special reports, and have received good social responses.
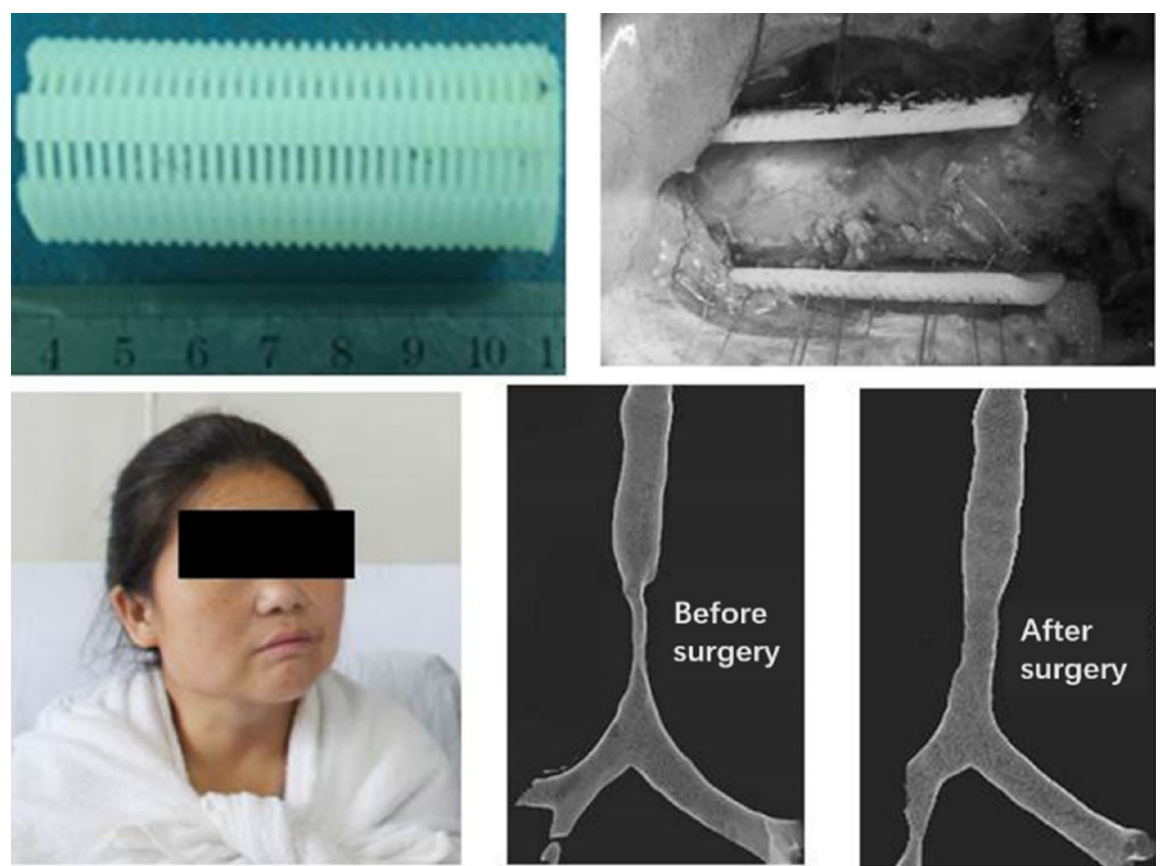

3D-printed biodegradable tracheal stent and its clinical application 


\section{Multi-nozzle 3D cell-printing technology}

The project focuses on the multi-cell in situ 3D printing technology, aiming for the biofabrication of multi-tissue such as vascular muscle. By taking the ink-jetting and extrusionbased 3D printing technologies as the core, a multi-nozzle $3 \mathrm{D}$ cell-printing equipment was developed in-house, for precise fabrication of soft tissue such as blood vessel, muscle or skin from the hydrogel-based composition of cells and bioac- tives via cross-linking. This has provided a novel solution for the challenges faced by tissue engineering for complex vascularization tissue construct and regeneration. The maximum printing size of the bespoke equipment is $100 \mathrm{~mm} \times$ $100 \mathrm{~mm} \times 100 \mathrm{~mm}$, providing sterile surgical condition for the in situ printing. In addition, the multi-nozzle system is adaptable for various forming mechanisms of multiple materials, providing coaxial nozzle, spray nozzle and single-tube nozzle, fulfilled the printing requirement of hollow tube gel and microfilament gel at the range of 400-1200 $\mu \mathrm{m}$ [26-34].

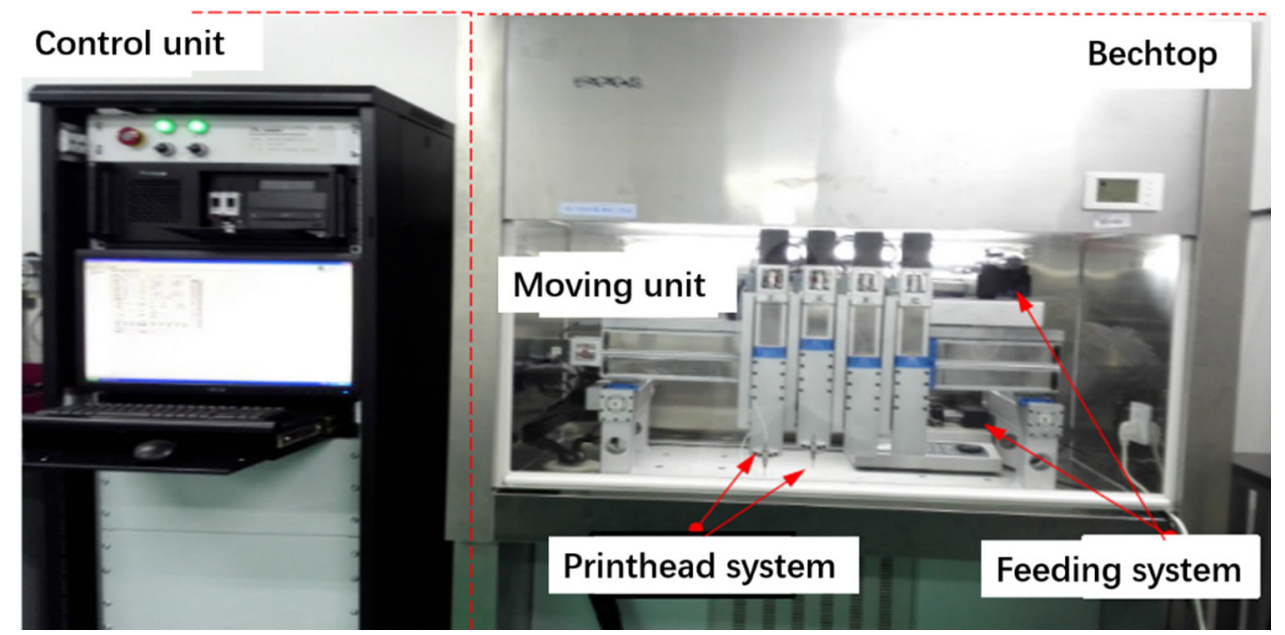

Bespoke multi-nozzle biological 3D cell-printing system

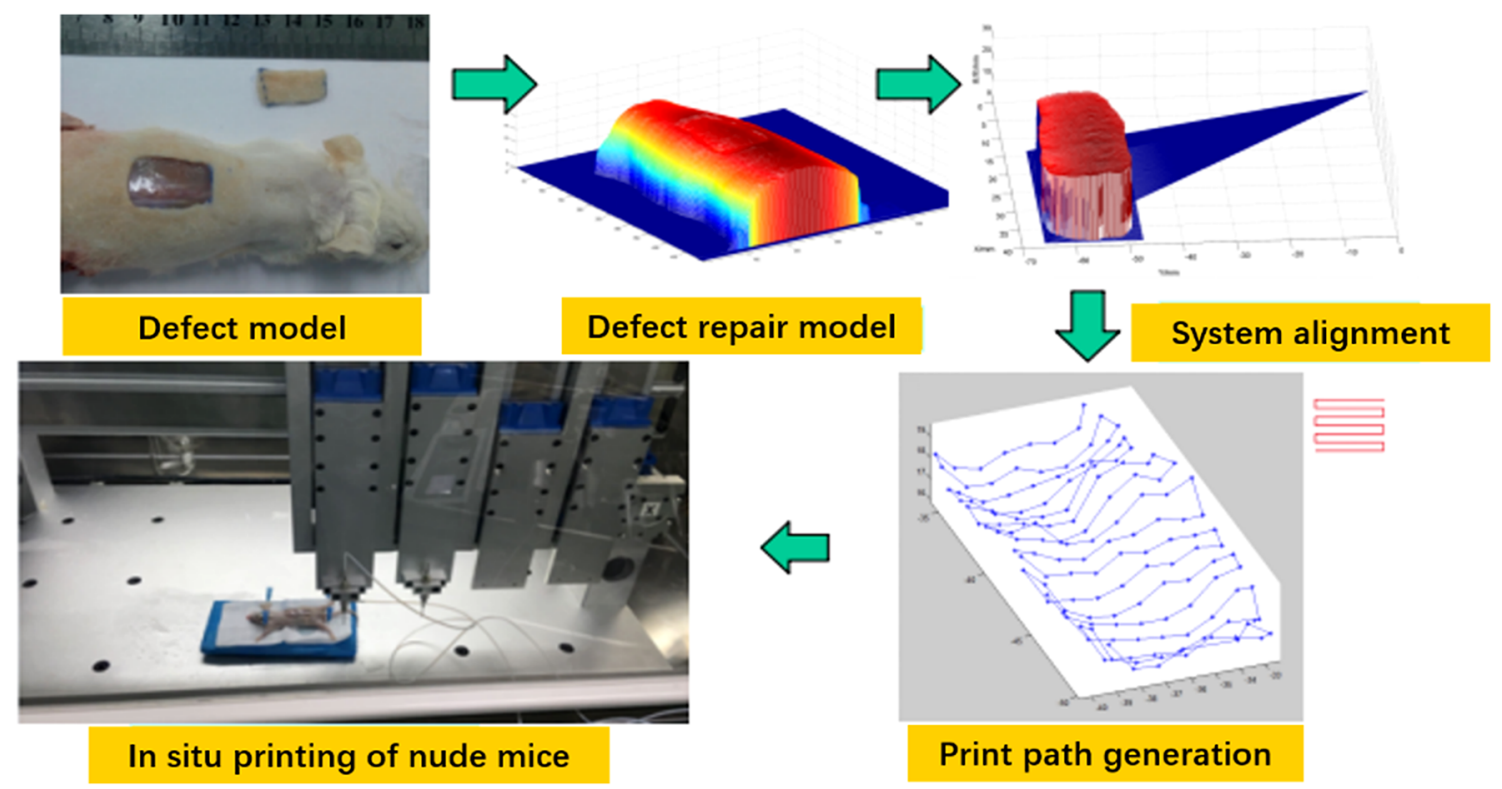

In situ cell-printing: the in-house developed equipment and animal test 


\section{Facilities}

The biofabrication laboratory has many facilities including cell and tissue culture facilities such as cell culture incubator and clean hood, many types of cell and tissue printing equipment such as ink-jetting cell printer, multi-material extrusion-based printer and laser printer, all printers are bespoke in-house by the members themselves, and the print- ing cell types cover skin, heart, muscle, ligament, cartilage and bone tissue. In addition, the laboratory has also many equipment for functional evaluation of the printed scaffolds and tissue such as Inverted fluorescence microscope, SEM, micro-CT, and mechanical testing equipment such as materials testing machine, fatigue testing machine, hip/knee joint simulator. Therefore, most of the functional evaluation work can be done within the laboratory conveniently and high efficiently with the help of many laboratory assistants.

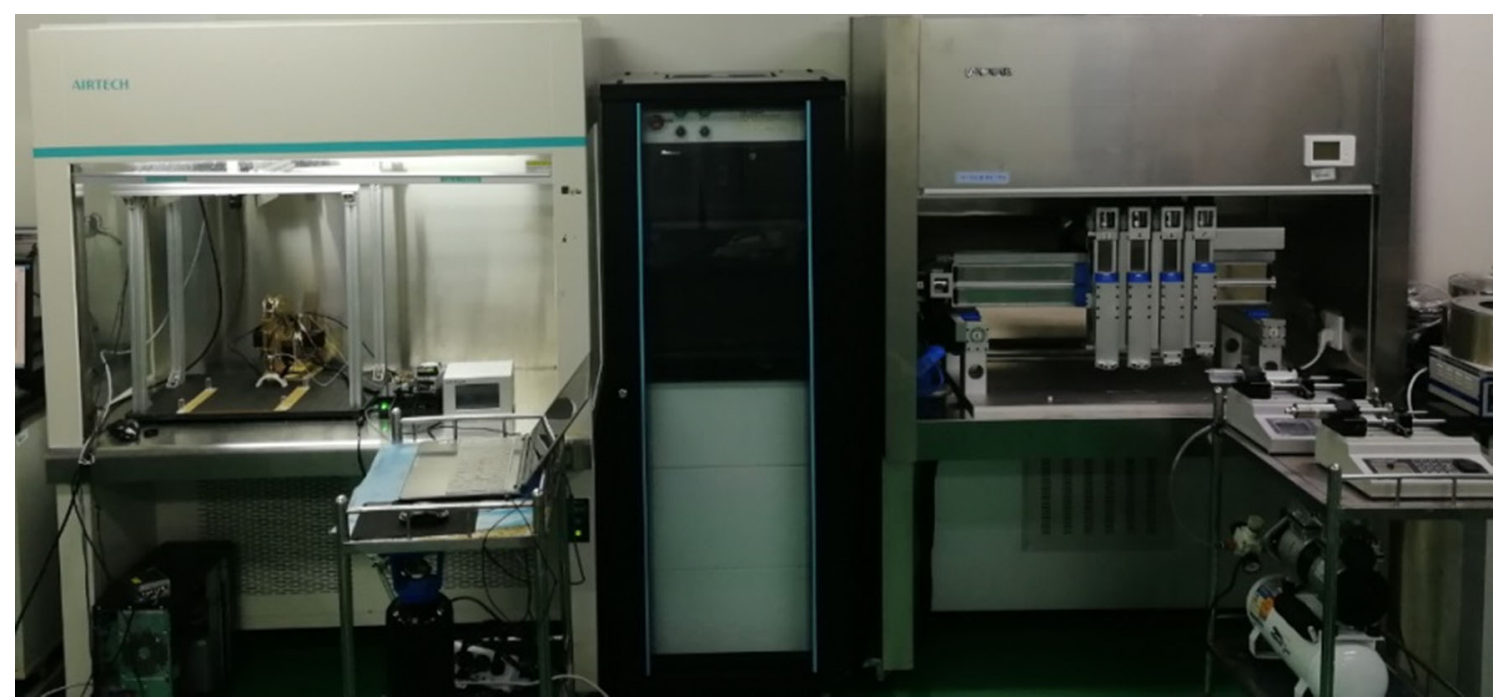

In situ cell-printing: the in-house developed equipment and animal test
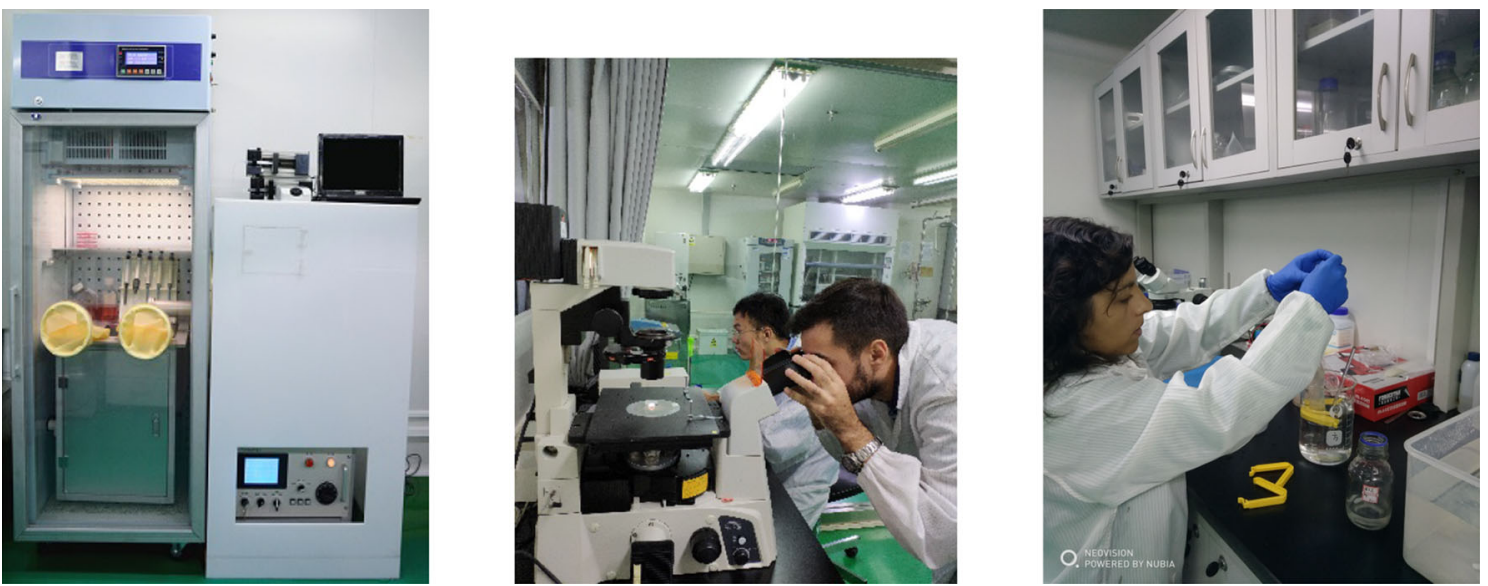

(a)Bespoke neuron culture and printer for brain-like tissue reconstruction; (b) cell culture room; (c) preparation room 

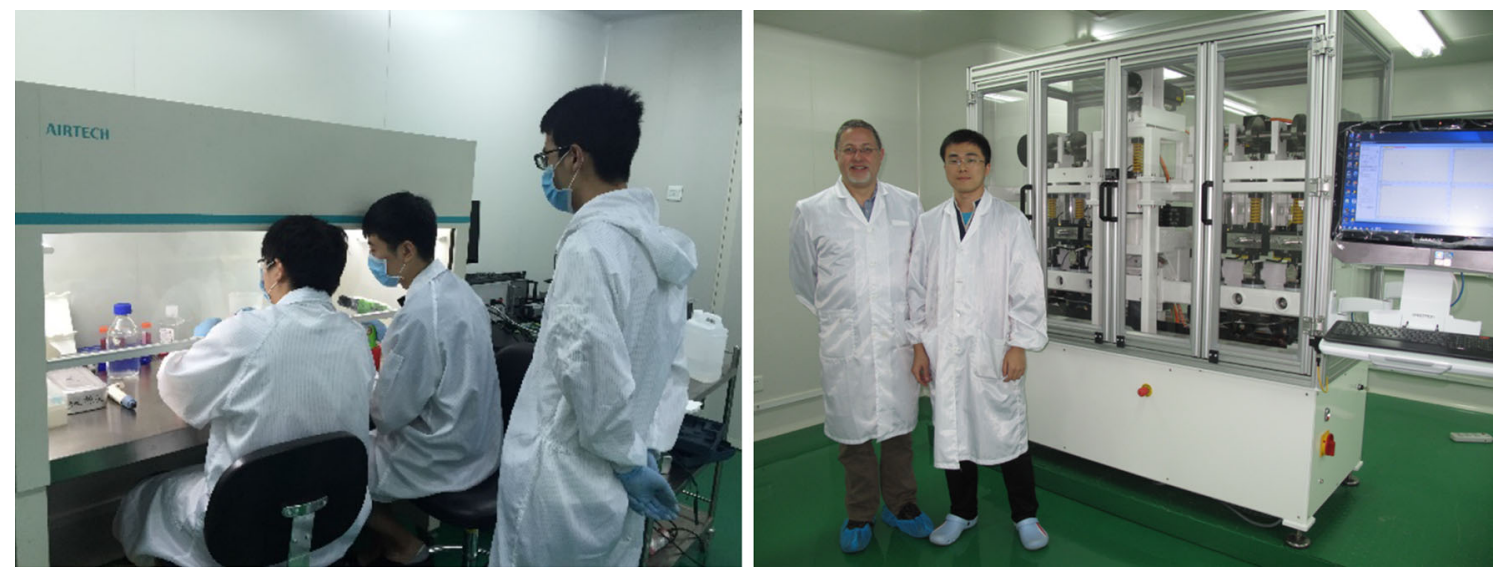

(a) Cell and tissue culture room; (b) hip simulator for hip joint wear test

\section{International collaboration}

The team has built solid foundation with many international research centers in the past decade, including Ph.D. student exchange, visiting scholars and international program. Research centers include the Federal Materials Testing Center of Germany (BAM) in Berlin, UCL Orthopaedic Bioengineering Research Group within the Division of Surgery and Interventional Science, University College London, Biomaterials and Biomechanics Research Group, University of Las Palmas de Gran Canaria (ULPGC), University of Minho in Porto, and Russian Academy of Science. Agreements of collaboration have been signed with each individual partner, and in total of 18 co-publications were achieved on the basis of the collaboration.

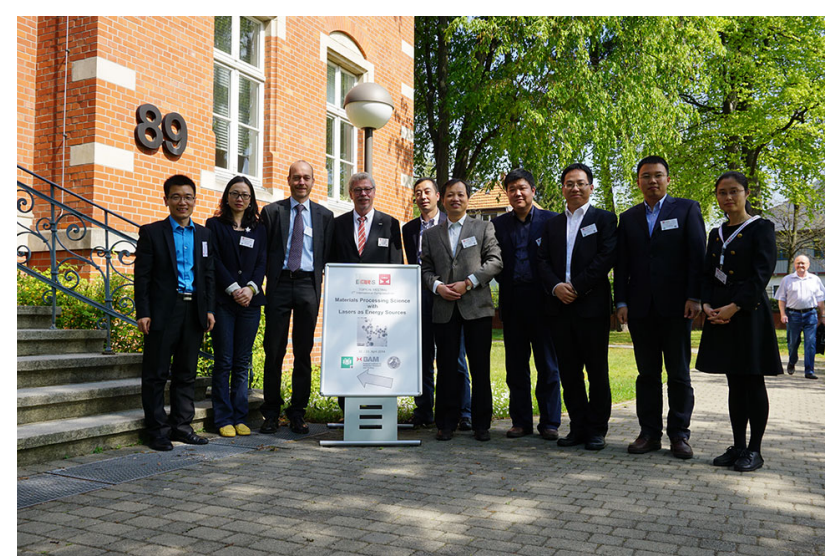

2014 BAM in Berlin, Germany

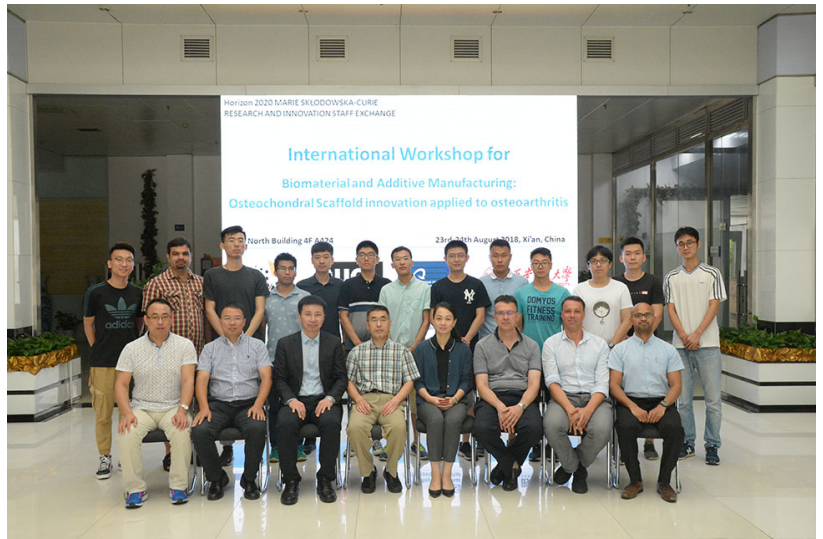

2018 BAMOS international workshop in Xi'an

\section{References}

1. Kang J, Wang L*, Yang C et al (2018) Custom design and biomechanical analysis of 3D printed PEEK rib prostheses. Biomech Model Mechanobiol 17(4):1083-1092

2. Qin M, Liu Y, Wang L et al (2015) Design and optimization of the fixing plate for customized mandible implants. J Cranio-Maxillofac Surg 43(7):1296-1302

3. Wang L, Yang W, Peng X et al (2015) Effect of progressive wear on the contact mechanics of hip replacements-does the realistic surface profile matter? J Biomech 48(6):1112-1118

4. Sun C, Wang L, Wang $Z$ et al (2015) Finite element analysis of a retrieved custom-made knee prosthesis. J Mech Med Biol 15(03):458-472

5. Dong LL, Guo PN, Liu R et al (2017) Three-dimensional printing technology facilitates customized pelvic prosthesis implantation in malignant tumor surgery: a case report. Int J Clin Exp Med 10(7):11020-11025

6. Wang L, Kang J, Sun C et al (2017) Mapping porous microstructures to yield desired mechanical properties for application in $3 \mathrm{D}$ printed bone scaffolds and orthopaedic implants. Mater Des 133(Supplement C):62-68 
7. Bian W, Li D, Lian Q et al (2012) Fabrication of a bio-inspired beta-Tricalcium phosphate/collagen scaffold based on ceramic stereolithography and gel casting for osteochondral tissue engineering. Rapid Prototyp J 18(1):68-80

8. Yi C, Li D, Jing W (2013) Using variable beam spot scanning to improve the efficiency of stereolithography process. Rapid Prototyp J 19(2):100-110

9. Zhang W, Lian Q, Li Det al (2014) Cartilage repair and subchondral bone migration using 3D printing osteochondral composites: a oneyear-period study in rabbit trochlea. Biomed Res Int 5:746138

10. Zhang W, Qin L, Li D et al (2015) The effect of interface microstructure on interfacial shear strength for osteochondral scaffolds based on biomimetic design and 3D printing. Mater Sci Eng, C 46:10-15

11. Lan PH, Li XK, Fan XL et al (2016) Ceramic coating of a titanium alloy implant prevents cartilage damage due to localized cartilage defects. J Biomater Tissue Eng 6(8):602-612

12. Wu X, Lian Q, Li D et al (2017) Tilting separation analysis of bottom-up mask projection stereolithography based on cohesive zone model. J Mater Process Technol 243:184-196

13. Qin L, Yang F, Xin H et al (2017) Oxygen-controlled bottom-up mask-projection stereolithography for ceramic 3D printing. Ceram Int 43(17):14956-14961

14. Chen X, Zhao G, Wu Y et al (2017) Cellular carbon microstructures developed by using stereolithography. Carbon 123:34-44

15. He J, Jiang N, Qin T et al (2017) Microfiber-reinforced nanofibrous scaffolds with structure and material gradients to mimic ligamentto-bone interface. J Mater Chem B 5(43):8579-8590

16. Blunn G, Tamaddon M, Liu C et al (2017) Intrinsic osteoinductivity of porous titanium scaffold for bone tissue engineering. Int $\mathbf{J}$ Biomater 2017:5093063

17. Mao M, He J, Liu Y et al (2012) Ice-template-induced silk fibroinchitosan scaffolds with predefined microfluidic channels and fully porous structures. Acta Biomater 8(6):2175-2184

18. He J, Wang Y, Liu Y et al (2013) Layer-by-layer micromolding of natural biopolymer scaffolds with intrinsic microfluidic networks. Biofabrication 5(2):025002

19. Li X, He J, Bian W et al (2014) A novel silk-based artificial ligament and tricalcium phosphate/polyether ether ketone anchor for anterior cruciate ligament reconstruction - safety and efficacy in a porcine model. Acta Biomater 10(8):3696-3704

20. He J, Qin T, Liu Y et al (2014) Electrospinning of nanofibrous scaffolds with continuous structure and material gradients. Mater Lett 137:393-397
21. He J, Zhang W, Liu Y et al (2015) Design and fabrication of biomimetic multiphased scaffolds for ligament-to-bone fixation. Mater Sci Eng, C 50:12-18

22. He J, Liu Y, Hao X et al (2012) Bottom-up generation of 3D silk fibroin-gelatin microfluidic scaffolds with improved structural and biological properties. Mater Lett 78(7):102-105

23. He J, Xia P, Li D (2015) Development of melt electrohydrodynamic 3D printing for complex microscale poly ( $\varepsilon$-caprolactone) scaffolds. Biofabrication 8(3):035008

24. Li X, He J, Zhang W et al (2016) Additive manufacturing of biomedical constructs with biomimetic structural organizations. Materials 9(11):909

25. Huang L, Wang L, He J et al (2016) Tracheal suspension by using 3-dimensional printed personalized scaffold in a patient with tracheomalacia. J Thorac Dis 8(11):3323-3328

26. Liu Y, Li X, Qu X et al (2012) The fabrication and cell culture of three-dimensional rolled scaffolds with complex microarchitectures. Biofabrication 4(1):015004

27. Zhao X, He J, Xu F et al (2016) Electrohydrodynamic printing: a potential tool for high-resolution hydrogel/cell patterning. Virtual Phys Prototyp 11(1):57-63

28. He J, Xu F, Cao Y et al (2016) Towards microscale electrohydrodynamic three-dimensional printing. J Phys D Appl Phys 49(5):055504

29. He J, Chen R, Lu Y et al (2016) Fabrication of circular microfluidic network in enzymatically-crosslinked gelatin hydrogel. Mater Sci Eng, C 59:53-60

30. Zhang B, He J, Li X et al (2016) Micro/nanoscale electrohydrodynamic printing: from 2D to 3D. Nanoscale 8(34):15376-15388

31. Qu XL, Xia P, He JK et al (2016) Microscale electrohydrodynamic printing of biomimetic PCL/nHA composite scaffolds for bone tissue engineering. Mater Lett 185:554-557

32. Li X, Lian Q, Li DC et al (2017) Development of a robotic arm based hydrogel additive manufacturing system for in situ printing. Appl Sci Basel 7(1):73

33. Mao M, He JK, Li X et al (2017) The emerging frontiers and applications of high-resolution 3D printing. Micromachines 8(4):113

34. He J, Xu F, Dong R et al (2017) Electrohydrodynamic 3D printing of microscale poly ( $\varepsilon$-caprolactone) scaffolds with multi-walled carbon nanotubes. Biofabrication 9(1):015007 